\title{
Relationship between apathy and tumor location, size, and brain edema in patients with intracranial meningioma
}

This article was published in the following Dove Press journal:

Neuropsychiatric Disease and Treatment

13 July 2015

Number of times this article has been viewed

\author{
Yihua Pengl,* \\ Chunhong Shao ${ }^{1, *}$ \\ Ye Gong ${ }^{2}$ \\ Xuehai Wu ${ }^{2}$ \\ Weijun Tang ${ }^{3}$ \\ Shenxun Shi' \\ 'Psychiatry Department, \\ ${ }^{2}$ Neurosurgery Department, \\ ${ }^{3}$ Radiology Department, Huashan \\ Hospital, Fudan University, People's \\ Republic of China \\ *These authors contributed equally \\ to this work
}

Correspondence: Shenxun Shi Psychiatry Department, Huashan Hospital, I2 Wulumuqi Road (Middle), Shanghai 200040, People's Republic of China

Tel +86 2l 52888153

Email drshishenxun@126.com
Background: The purpose of this study is to assess the relationship between apathy and tumor location, size, and brain edema in patients with intracranial meningioma.

Methods: We enrolled 65 consecutive patients with meningioma and 31 normal controls matched for age, gender, and education. The patients were divided into frontal or non-frontal (NF) meningioma groups based on magnetic resonance imaging; the frontal group was then subdivided to dorsolateral frontal (DLF), medial frontal (MF), and ventral frontal (VF) groups. Tumor size and brain edema were also recorded. Apathy was assessed by the Apathy Evaluation Scale (AES). Assessments were carried out 1 week before and 3 months after surgery, respectively. Logistic regression analysis was performed to identify the predictive effect of tumor size, location, and brain edema on apathy. Analysis of variance and chi-square analysis were applied to compare apathy scores and apathy rates among the frontal, NF, and normal control groups, and all subgroups within the frontal group.

Results: Compared with the NF and control groups, the mean AES score was much higher in the frontal group (34.0 \pm 8.3 versus $28.63 \pm 6.0, P=0.008$, and 26.8 $\pm 4.2, P<0.001$ ). Subgroup analysis showed that AES scores in the MF group (42.1 \pm 6.6$)$ and VF group (34.7 \pm 8.0$)$ were higher than in the DLF group (28.5 \pm 4.36$)$, NF group, and control group $(P<0.05)$. The apathy rate was $63.6 \%$ in the MF group and $25 \%$ in the VF group, and significantly higher than in the DLF (5.6\%), NF $(5.3 \%)$, and control $(0 \%)$ groups $(P<0.001)$. A moderate correlation was found between AES score and mean diameter of the meningioma in all patient groups. Further analysis demonstrated that the correlation existed in the DLF ( $r=0.52, P=0.032), \operatorname{MF}(r=0.84, P<0.001)$, and VF $(r=0.64$, $P=0.008)$ groups, but not in the NF group $(r=0.19, P=0.448)$. The AES score was much higher in patients with brain edema than in those without brain edema ( $34.73 \pm 8.28$ versus $28.77 \pm 4.20$, $t=3.545, P=0.001)$. In subgroups within frontal meningioma patients, the statistical significance above only existed in the MF group ( $43.50 \pm 5.26$ versus $25.67 \pm 6.03, P=0.001)$. Also, we examined the effect of related factors, such as age, sex, education, tumor size, tumor location and edema on the occurrence of apathy. The binary logistic regression analysis showed that $\mathrm{MF}[P=0.023$, $\operatorname{Exp}(B)=145.6]$ and size $[P=0.012, \operatorname{Exp}(B)=1.20]$ got into the regression equation. Thirty-two patients underwent follow-up post-surgery. A significant reduction in AES was found in the MF group (AES1 - AES2 $=6.86 \pm 6.82, t=2.68, P=0.04$ ), but not in any of the other groups.

Conclusion: Apathy occurs frequently in patients with frontal meningioma, and is more severe, especially in the MF region. Apathy is probably correlated with tumor location and size. Brain edema might increase the severity of apathy.

Keywords: apathy, Apathy Evaluation Scale, meningioma, edema, location, size

\section{Introduction}

Apathy, defined by Marin as a neuropsychiatric syndrome due to lack of motivation, ${ }^{1}$ could present in several diseases, including schizophrenia, Alzheimer's disease, 
Parkinson's disease, and stroke, and with brain injury and brain tumors. ${ }^{2-9}$ Described as having a lack of interest and concern, being indifferent, and finding it hard to initiate and sustain goal-directed behavior, these patients present disability of different degrees in both self-care and social activity in terms of severity of apathy.

Neurologists and psychiatrists have attempted over many years to clarify the pathological mechanism of apathy. In recent times, a large number of studies have demonstrated that apathy is related to abnormal changes in the frontal lobe. ${ }^{4,10-12}$ These have mainly been cross-sectional studies in patients with Alzheimer's disease or Parkinson's disease. Using advanced imagining techniques, researchers have detected abnormalities in several frontal areas in patients with apathy when compared with those without apathy. However, it is not sufficient to conclude that apathy is a result of destruction or dysfunction of the frontal area, because these studies could not answer whether apathy was reversible when the frontal lesions were cured, as the pathology is irreversible in most of the disease ever used to study apathy.

Much less attention has been focused on apathy in patients with brain tumors, while a few studies on meningioma may give light to further study of apathy. There have been a few case reports and some clinical research on patients with meningioma located at the midline or base of the frontal lobe in whom typical symptoms of apathy have been recorded. ${ }^{7,11}$ Interestingly, it was also reported that these symptoms were completely or partially relieved after tumor resection. These findings are likely to tell that apathy could be result from compressed specific frontal region, eg, medial frontal (MF) or orbital frontal, caused by meningioma. In that case, this pathological process is reversible. If this is true, it will be important to understand the etiology of apathy, and it might provide a new disease model for further study. However, case reports are not powerful evidence, and lack of controls and validated tools to measure apathy are common shortcomings in the limited amount of clinical research done so far. Further, although a relationship between apathy and tumor location has been mentioned, it has not been investigated in depth in previous studies.

With the above considerations in mind, we used the Apathy Evaluation Scale (AES) to assess and compare the severity of apathy according to location, size and edema of the tumor in the brain. The severity of apathy before and after surgery was also compared.

\section{Materials and methods}

\section{Participants}

The study comprised all consecutive patients who underwent surgery for meningioma at Huashan Hospital, Fudan
University, from March 3, 2011 to December 11, 2011. Approval to undertake this research was received from the human research ethics committee at Huashan Hospital. Normal controls matched for age, sex, and education were recruited from patients' families or relatives. Inclusion criteria were: ipsilateral meningioma diagnosed by magnetic resonance imaging (MRI) prior to surgery; and Chinese as the mother language and an education level of primary school or above, so that patient questionnaires could be understood. Exclusion criteria were: multiple meningioma or tumor invading two or more lobes; meningioma located in the ventricles and invading the basal ganglia or in the posterior cranial fossa; inability to self-care due to severe physical illness or disability; concomitant schizophrenia, bipolar disorder, or severe depressive disorder; antipsychotic, antidepressant, or sedative medication due to mental illness within the previous 3 months; alcohol or drug abuse; history of a disease of the central nervous system; and severe brain injury within the previous year.

\section{MRI}

An MRI scan was performed on at least two occasions, ie, before and 3 months after surgery. T1, T2, flair, and enhanced series were obtained. Tumor location was judged according to the enhanced series. Tumor size was recorded as the mean diameter, which is the cube root of the product of the maximum transverse, vertical, and horizontal diameters measured in the enhanced series. Brain edema was judged by the T2 and flair series. The MRI scan 3 months after surgery was used to exclude cases with complications such as hydrocephalus or tumor recurrence.

\section{Measures}

Apathy was assessed using the AES (clinician version). This scale has 18 items, each rated from 1 to 4 . It generates emotional, cognitive, and behavioral apathy subscale scores together with a full rated score of 18-72, with higher scores reflecting increasing severity of apathy. It can be self-rated or scored via an informant or a clinician. In this study, the AES was rated by a clinician. The AES has been widely validated, and shown good reliability and validity in several clinical populations. ${ }^{8}$ The International Classification of Diseases, 10 th Revision was used to screen for other psychiatric illness.

Participants also underwent standardized emotional evaluation (Self-Rating Anxiety Scale, Self-Rating Depression Scale) and cognitive assessment (Mini-Mental State Examination, Wisconsin Card Sorting Test, Iowa Gambling Test, DigitalSpan). The emotional and cognitive assessment data will be reported elsewhere. 


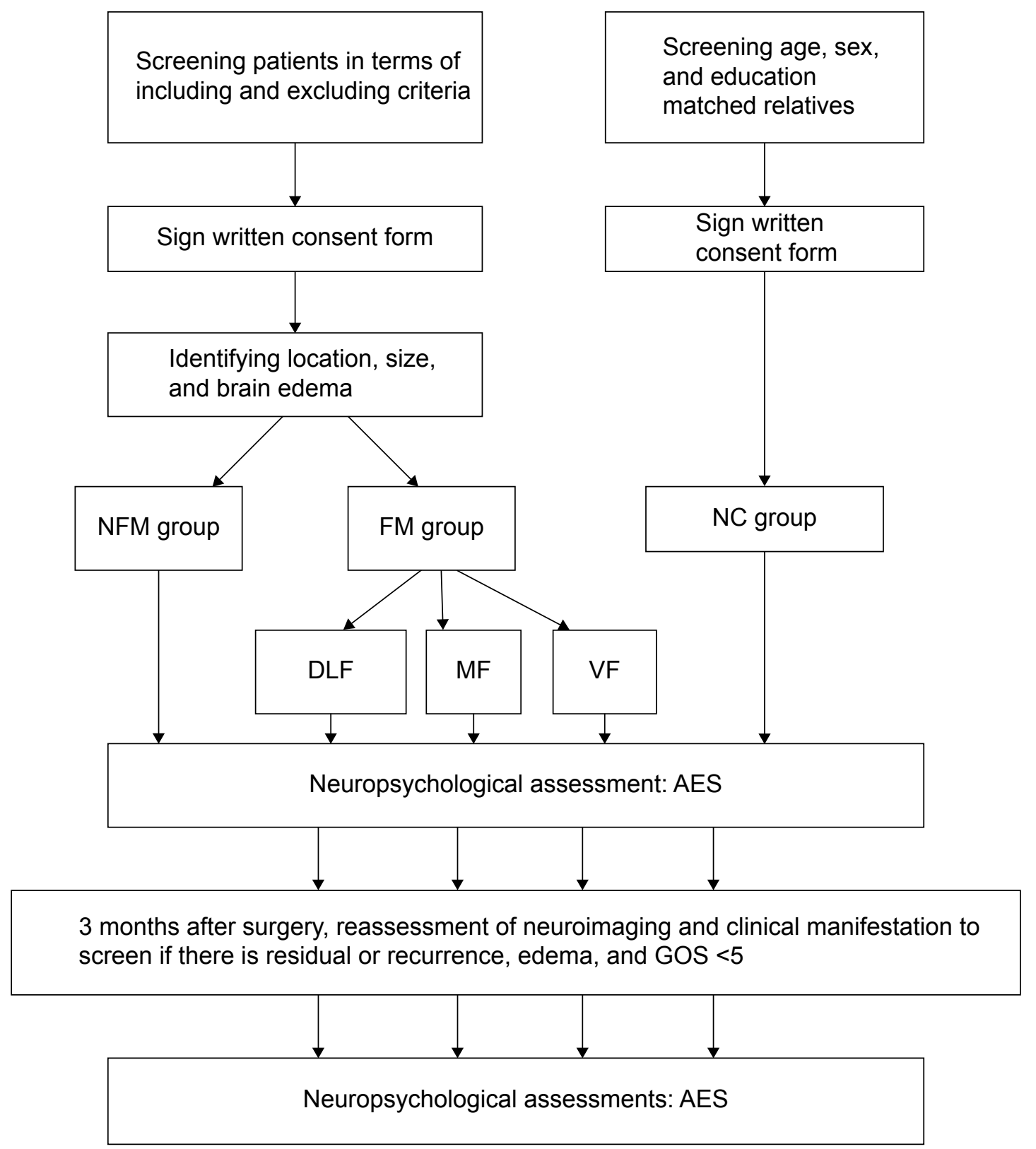

Figure I Flowchart showing how participants were recruited and grouped.

Abbreviations: NFM, non-frontal meningioma; FM, frontal meningioma; NC, normal control; DLF, dorsolateral frontal; MF, medial frontal; VF, ventral frontal; AES, Apathy Evaluation Scale; GOS, Glasgow Outcome Scale.

Sixty-seven patients with meningioma were enrolled, 65 of whom completed all of the assessments. Thirty-seven normal controls matched for age, sex, and education were recruited from the patients' families or relatives. The recruitment and assessment process is shown in Figure 1.

\section{Results}

\section{Patient demographic and clinical characteristics}

The age, sex, and education distribution for all participants is shown in Table 1 . There was no statistically significant difference in age, sex, and education between the groups. Tumor size and edema were recorded for all patients Table 2).

\section{AES results}

AES scores before surgery were obtained for all 65 patients and 37 controls. Only one patient in the non-frontal (NF) meningioma group showed symptoms of apathy versus 12 in the frontal meningioma group. Not surprisingly, there were no symptoms of apathy in the normal control group (Table 3). 
Table I Demographic descriptions in all groups of participants

\begin{tabular}{|c|c|c|c|c|c|c|}
\hline & \multicolumn{3}{|l|}{ FM } & \multirow[t]{2}{*}{ NF } & \multirow[t]{2}{*}{ NC } & \multirow[t]{2}{*}{$P$-value } \\
\hline & DLF & VF & MF & & & \\
\hline Participants (n) & 18 & 16 & II & 19 & 37 & \\
\hline Gender (M/F) & $2 / 16$ & $8 / 8$ & $3 / 8$ & $6 / 13$ & $12 / 25$ & $0.214^{x}$ \\
\hline Age (years), mean $\pm S D$ & $53.1 \pm 5.9$ & $52.9 \pm 8.3$ & $51.2 \pm 10.2$ & $48.4 \pm 14.9$ & $46.1 \pm 11.4$ & $0.165^{A}$ \\
\hline Education & & & & & & $0.194^{x}$ \\
\hline Primary school & 4 & 5 & 4 & 2 & 8 & \\
\hline Junior school & 7 & 6 & 5 & 4 & 14 & \\
\hline Senior school & 5 & 5 & 1 & 10 & 12 & \\
\hline University & 2 & 0 & 1 & 3 & 3 & \\
\hline
\end{tabular}

Notes: ${ }^{\times}$Chi-squared test; ${ }^{A}$ one-way analysis of variance test.

Abbreviations: FM, frontal meningioma; DLF, dorsolateral frontal; VF, ventral frontal; MF, medial frontal; NF, non-frontal; NC, normal control; M, male; F, female; SD, standard deviation.

\section{Relationship between apathy and location of meningioma}

AES scores were much higher in the MF group than in the other groups $(F=8.766, d f=4, P=0.000)$. The second highest AES score was in the ventral frontal (VF) group. AES scores were much lower in the other groups. However, when compared with the normal control group, there was no statistically significant difference in AES scores between the dorsolateral frontal (DLF) and NF groups. The apathy rate was statistically different among all studied groups (Figure 2, Table 3).

\section{Relationship between tumor size and AES}

There was a correlation between AES scores and tumor size in patients with frontal meningioma. Compared with the DLF group ( $r=0.52, P=0.032$, Figure $3 \mathrm{~A}$ ), a stronger correlation was found in the VF group $(r=0.64, P=0.008$, Figure $3 \mathrm{C})$ and the MF group ( $r=0.84, P<0.001$, Figure 3B). No correlation was found between AES and tumor size in the group with NF meningioma ( $r=0.19, P=0.448$, Figure 3D).

\section{Relationship between brain edema and AES}

AES scores were higher in patients with brain edema $(34.73 \pm 8.28$ versus $28.77 \pm 4.20, P<0.01)$ than in those without brain edema. Further analysis showed a higher AES score in MF patients with edema than in those without edema $(P<0.05)$. Although higher AES scores were found in
DLF and VF patients with edema than in their counterparts without edema, the difference was not statistically significant $(P>0.05)$. Further, there was no difference in AES scores between those with and without brain edema in the NF meningioma group (Figure 4, Table 4).

\section{Relationship between tumor size, location, brain edema, and AES}

Using regression analysis, we examined that which factors were probably related to apathy, such as age, sex, education, tumor size, location (DLF, MF, VF, NF), and brain edema. The binary logistic regression analysis showed that MF $[P=0.023, \operatorname{Exp}(\mathrm{B})=145.6]$ and size $[P=0.012, \operatorname{Exp}(\mathrm{B})=1.20]$ got into the regression equation.

\section{Comparison of AES scores before and after surgery}

Thirty-four patients returned for follow-up 3 months after surgery, the other patients were either unwilling to continue or unable to be contacted. Two of these 34 patients were excluded from the study because of tumor recurrence or incomplete tumor resection confirmed on MRI. The distribution of the remaining 32 patients is shown in Table 5.

We compared the difference in AES scores before and after surgery using the paired $t$-test. Although changes were found in AES scores between the MF and VF groups before and after surgery, the difference was only statistically significant in the MF group $(P<0.01)$. Changes in AES scores

Table 2 Brain tumor size and edema in the patient groups

\begin{tabular}{llllll}
\hline & DLF & MF & VF & NF & P-value \\
\hline MD (mm), mean \pm SD & $32.53 \pm 10.49$ & $39.69 \pm 13.23$ & $33.15 \pm 14.07$ & $33.41 \pm 16.51$ & $0.533^{A}$ \\
With/without edema & $8 / 9$ & $7 / 4$ & $9 / 7$ & $8 / 11$ & $0.404^{\times}$ \\
\hline
\end{tabular}

Notes: ${ }^{\times}$Chi-squared test; ${ }^{A}$ one-way analysis of variance test.

Abbreviations: DLF, dorsolateral frontal; VF, ventral frontal; MF, medial frontal; NF, non-frontal; MD, mean diameter; SD, standard deviation. 
Table 3 Mean score and apathy rates in all groups before surgery

\begin{tabular}{|c|c|c|c|c|c|c|}
\hline & \multicolumn{3}{|l|}{ Frontal* } & \multirow[t]{2}{*}{ NF } & \multirow[t]{2}{*}{ Control } & \multirow[t]{2}{*}{$P$-value } \\
\hline & DLF & MF & VF & & & \\
\hline AES score, mean \pm SD & $28.5 \pm 4.36$ & $42.1 \pm 6.6^{\mathrm{a}, \mathrm{b}}$ & $34.7 \pm 8.0^{\mathrm{a}, \mathrm{c}}$ & $28.63 \pm 6.0$ & $26.8 \pm 4.2$ & $0.000^{A}$ \\
\hline Apathy $(\mathrm{Y} / \mathrm{N})$ & $1 / 17$ & $7 / 4$ & $4 / 12$ & $1 / 18$ & $0 / 37$ & $0.000^{x}$ \\
\hline Rate of apathy (\%) & 5.6 & 63.6 & 25.0 & 5.3 & 0 & \\
\hline
\end{tabular}

Notes: *Mean AES score for the whole frontal group is $34.0 \pm 8.3$, which is significantly higher than in the control $(P<0.01)$ and NF $(P<0.05)$ groups. Rate of apathy in the frontal group is $26.7 \%$ (I2/45), which is higher than in the control and NF groups $(P<0.05)$. ${ }^{\mathrm{P}} P<0.0$ I versus control; ${ }^{\mathrm{b}} P<0.01$ versus $N F$; ${ }^{\mathrm{c}} P<0.05$ versus $N F$ group; ${ }^{A}$ one-way analysis of variance test; ${ }^{\times}$chi-squared test.

Abbreviations: AES, Apathy Evaluation Scale; DLF, dorsolateral frontal; VF, ventral frontal; MF, medial frontal; NF, non-frontal; SD, standard deviation.

before and after surgery in the DLF and NF groups were not statistically significant (Figure 5, Tables 5 and 6).

\section{Discussion}

\section{Apathy and tumor location}

Studies of apathy in patients with meningioma are rare. One of the reasons for this is that neurosurgeons might not be familiar with the symptoms of apathy, so that mild apathy may go unrecognized or severe apathy may be treated as depression or dementia. ${ }^{13}$ Using the AES, we found that apathy is common in patients with frontal meningioma. Compared with normal controls and patients with NF meningioma, higher AES scores and apathy rates were found in patients with frontal meningioma, indicating that damage to the frontal lobe plays an important role in the etiology of apathy. This is similar to the conclusions of studies in Alzheimer's disease and Parkinson's disease. , $^{410-12}$

Further analysis of the frontal meningioma subgroups showed that apathy was most severe in the MF group, followed by the VF group, with the DLF group not showing

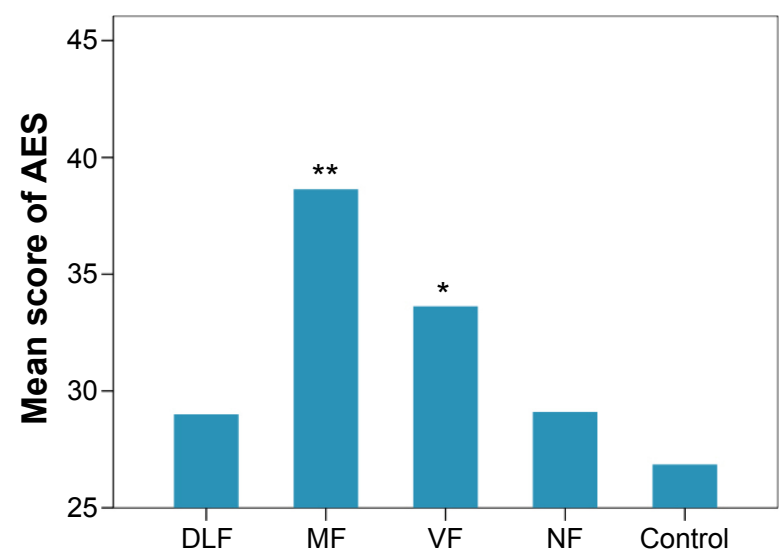

Figure 2 Mean AES scores in the patient groups before surgery and in the control group at baseline.

Notes: Mean AES scores are significantly higher in the MF and VF groups than in the NF and control groups. ${ }^{* * P}<0.0 \mathrm{I}$ versus the NF and control group, $* P<0.05$ versus the control and NF groups.

Abbreviations: AES, Apathy Evaluation Scale; DLF, dorsolateral frontal; VF, ventral frontal; MF, medial frontal; NF, non-frontal. any susceptibility to apathy. In regression analysis, MF was the most powerful independent risk factor for apathy, which underscores the importance of tumor location when considering the pathological mechanism of apathy.

The MF group mostly had parafalx meningioma, which compresses the medial aspect of the frontal lobe. It is not uncommon to see apathy when the MF lobe is damaged. In experiments done in monkeys, injury to the MF lobe caused a significant reduction in spontaneous behavior. ${ }^{14}$ Kang et al reported that an apathy rate of $43 \%$ in 100 patients with infarction of the anterior cerebral artery, which provides blood to the MF area. ${ }^{15}$ Therefore, we suspect that damage to structures within the MF, such as the anterior cingulate gyrus or corpus callosum, may be responsible for apathy. There is quite a lot of evidence showing the anterior cingulate cortex to be a key structure in generation of motivation ${ }^{16,17}$ and that it is somehow abnormal in patients with apathy. ${ }^{11,12,14}$ As we already know, the anterior cingulate cortex accepts dopaminergic projections from the nucleus accumbens, and plays an important role in the "reward system" ${ }^{18}$ It also connects widely with the limbic system and the frontal cortex, which is responsible for regulation of affect and for executive function. ${ }^{19}$ Compression of the anterior cingulate cortex by meningioma may cause a reduction in motivation, as well as in emotional arousal and execution of goal-directed behavior. There is also evidence indicating that damage to the corpus callosum, another important structure affected in this group, may be related to apathy. ${ }^{20,21}$ Although the corpus callosum was not thought to be involved in the network regulating motivation in a previous study, ${ }^{22}$ further research is needed to elucidate the underlying mechanism.

The VF group contained a majority of olfactory groove meningioma that compressed the VF. Bassiouni et al reported that $55 \%$ of their patients with this type of meningioma presented a number of psychiatric symptoms, of which $23 \%$ was apathy ${ }^{23}$ Combined with our current findings, it appears that the VF cortex is implicated in apathy. Atrophy in the orbital frontal cortex is a classical finding on imaging in patients with frontotemporal dementia, and most 
A

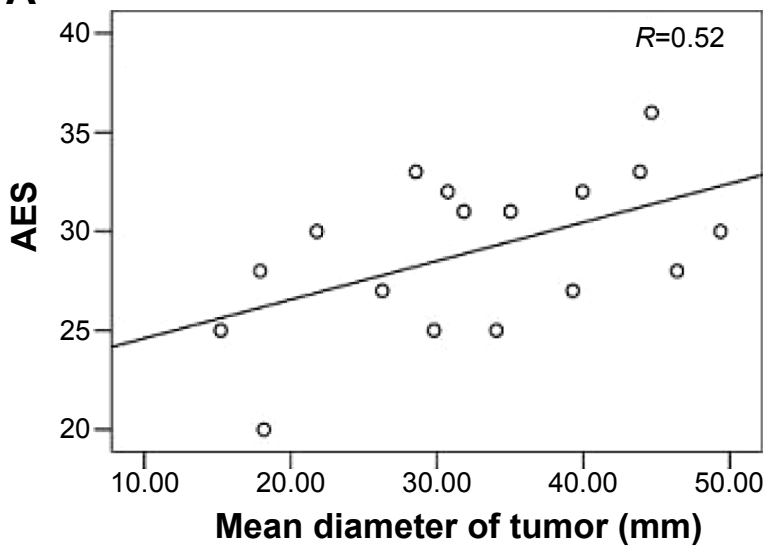

C

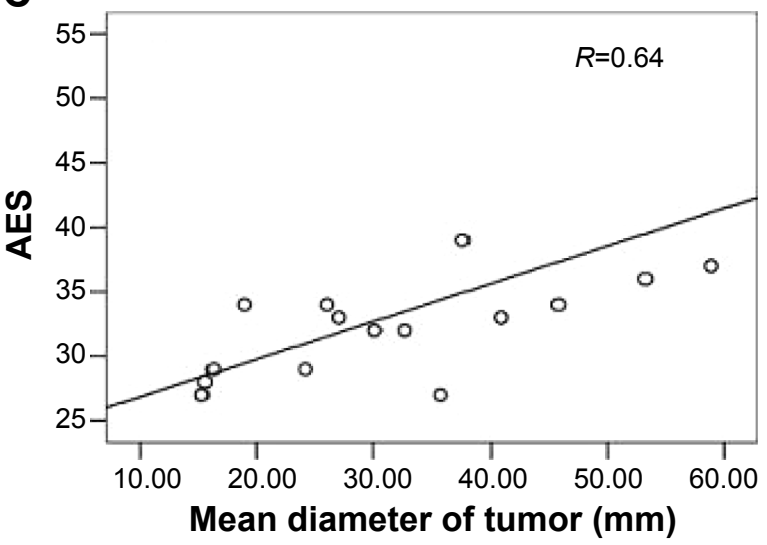

B

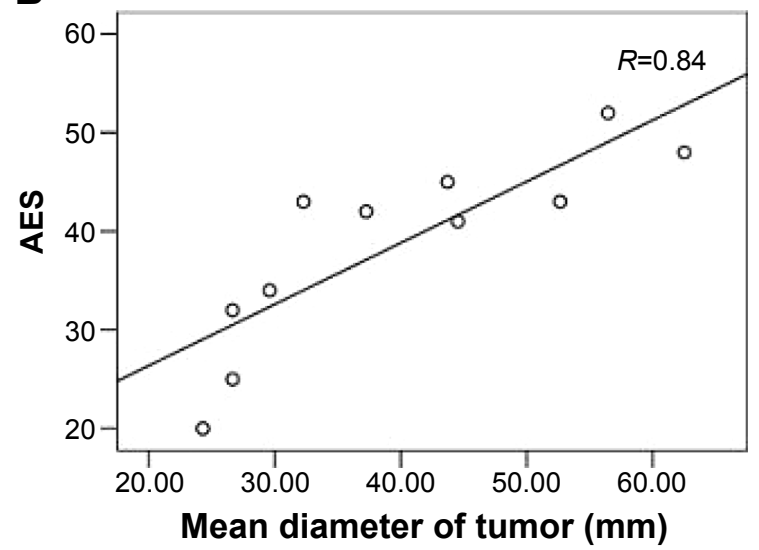

D

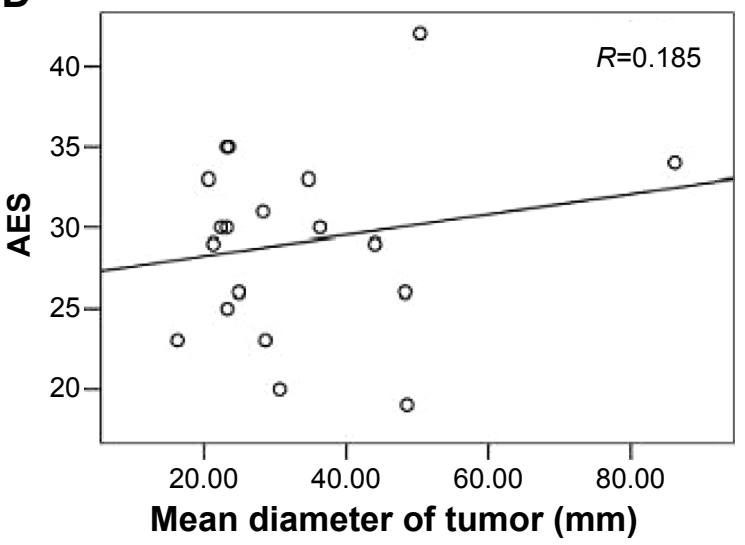

Figure 3 Correlations between mean tumor diameter and AES scores in all patient groups.

Notes: (A) There was a moderate correlation between AES score and tumor size in the DLF group $(r=0.52, P=0.032)$. (B) A strong correlation was found between AES score and tumor size in the MF group $(r=0.84, P<0.001)$. (C) A moderate correlation was found between AES score and tumor size in the VF group $(r=0.64, P=0.008)$. ( $D$ ) There was no correlation between AES score and tumor size in the NF group $(r=0.19, P=0.448)$.

Abbreviations: AES, Apathy Evaluation Scale; DLF, dorsolateral frontal; MF, medial frontal; VF, ventral frontal; NF, non-frontal.

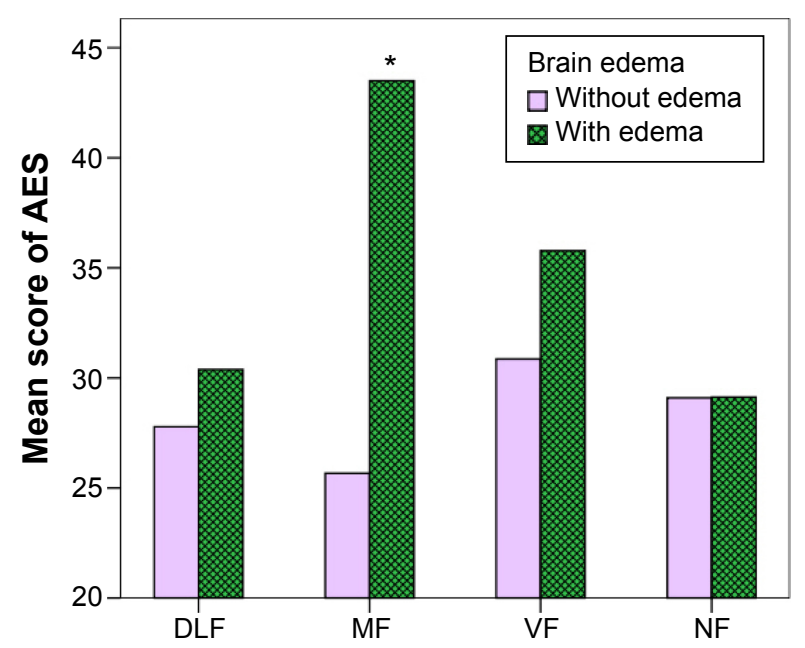

Figure 4 Comparison of mean AES scores between patients with and without edema for all groups.

Notes: Mean AES scores were higher in patients with brain edema than in those without brain edema in the DLF, MF, and VF groups. However, statistical significance was only found in the comparison within the MF group. $(* P<0.05)$. No obvious difference was found for such comparison within the NF group.

Abbreviations: AES, Apathy Evaluation Scale; DLF, dorsolateral frontal; VF, ventral frontal; MF, medial frontal; NF, non-frontal. of these patients have symptoms of apathy. However, in the current study we did not found VF is related to apathy, which might be attributed to our relatively small sample size. Our current results also suggest that damage to the VF might pose lower contribution to apathy compared with the effect of MF on apathy. In fact, large VF meningiomas can affect the ventral portion of the anterior cingulate cortex. ${ }^{24}$ This might also partly explain why patients in VF group presented apathy, though it was not as severe as that in MF group.

Table 4 Comparison of AES in patients with and without brain edema

\begin{tabular}{lllll}
\hline & DLF & VF & MF & NF \\
\hline $\begin{array}{l}\text { With edema } \\
\text { (AES), mean } \pm \text { SD }\end{array}$ & $30.38 \pm 3.54$ & $35.78 \pm 7.79$ & $43.50 \pm 5.26$ & $29.13 \pm 7.57$ \\
$\begin{array}{l}\text { Without edema } \\
\text { (AES), mean } \pm \text { SD }\end{array}$ & $27.78 \pm 4.01$ & $30.86 \pm 2.85$ & $25.67 \pm 6.03$ & $29.09 \pm 4.37$ \\
$t$-value & 1.397 & 1.580 & 4.839 & 0.012 \\
$P$-value & 0.183 & 0.136 & 0.001 & 0.990 \\
\hline
\end{tabular}

Abbreviations: AES, Apathy Evaluation Scale; DLF, dorsolateral frontal; VF, ventral frontal; MF, medial frontal; NF, non-frontal; SD, standard deviation. 


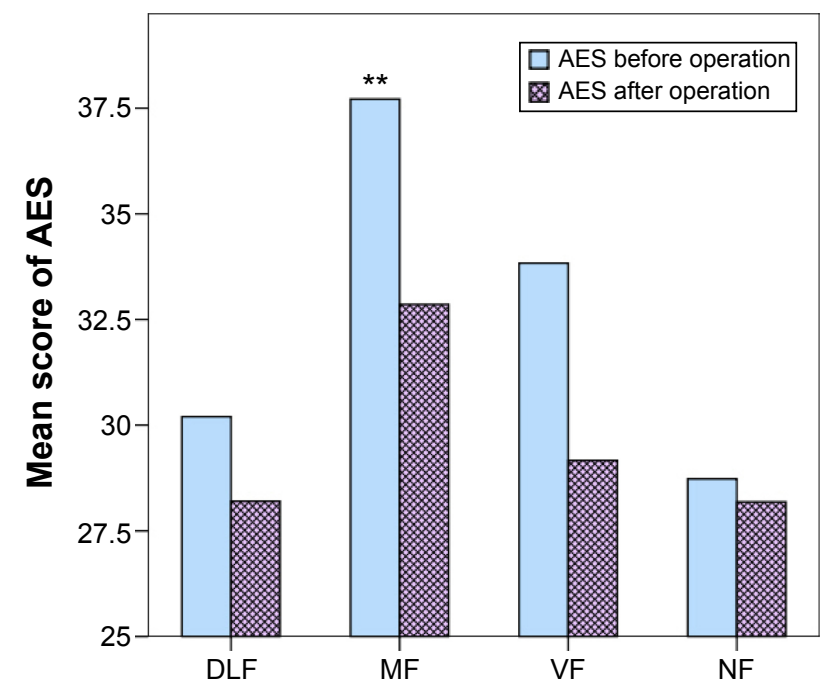

Figure 5 Comparison of mean AES scores before and 3 months after surgery for all groups.

Note: The AES score for patients in the MF group was significantly lower 3 months after surgery than before surgery $(* * P<0.0 I)$, but not in the DLF, VF, and NF groups. Abbreviations: AES, Apathy Evaluation Scale; DLF, dorsolateral frontal; VF, ventral frontal; MF, medial frontal; NF, non-frontal.

\section{Apathy and tumor size}

The present study demonstrated that, overall, AES scores were positively correlated with the mean tumor diameter, ie, AES scores increased with increasing tumor size. Typically, tumor size can indirectly reflect the degree of injury of peripheral nerve tissues due to mechanical damage and secondary ischemia. It is well known that the larger the tumor, the greater pressure the peripheral nerve tissue gets. Bassiouni et al found that patients with meningioma affecting the olfactory groove initially only presented symptoms of behavioral disinhibition, and then symptoms of apathy with time ${ }^{23}$ suggesting that apathy is associated with the chronicity of a brain tumor.

When comparing subgroups, we also demonstrated that the correlation between AES and tumor size existed in the MF group but not in the NF group. The reason for this probably relates to the location of the tumor. NF meningioma usually involves the temporal, parietal, and occipital lobes and the cerebellum. Typically, these brain regions are not involved in development of apathy. Because larger tumors cause more pervasive structural damage in the

Table 5 Patients with apathy before and after surgery

\begin{tabular}{lllll}
\hline & DLF & MF & VF & NF \\
\hline Total number & 9 & 7 & 6 & 10 \\
Apathy before surgery $(\mathrm{n})$ & 0 & 5 & 2 & $\mathrm{I}$ \\
Apathy after surgery $(\mathrm{n})$ & 0 & 2 & $\mathrm{I}$ & $\mathrm{I}$ \\
\hline
\end{tabular}

Abbreviations: DLF, dorsolateral frontal; MF, medial frontal; VF, ventral frontal; $\mathrm{NF}$, non-frontal.
Table 6 Changes in AES before and after surgery

\begin{tabular}{lllll}
\hline Group & AES2 & AESI - AES2 & $t$-value & $P$-value \\
\hline DLF & $28.33 \pm 5.20$ & $1.17 \pm 5.49$ & 0.08 & 0.94 \\
MF & $34.14 \pm 4.10$ & $6.86 \pm 6.82$ & 2.68 & 0.04 \\
VF & $29.17 \pm 3.43$ & $3.67 \pm 7.17$ & 1.24 & 0.27 \\
NF & $27.20 \pm 4.00$ & $0.50 \pm 6.15$ & 0.26 & 0.80 \\
\hline
\end{tabular}

Note: Data are presented as mean \pm standard deviation.

Abbreviations: AES, Apathy Evaluation Scale; AESI, AES assessment before surgery; $A E S 2, A E S$ assessment after surgery; DLF, dorsolateral frontal; MF, medial frontal; VF, ventral frontal; NF, non-frontal.

frontal lobe, frontal meningioma affects structure related to motivation. If a larger tumor locates in the DLF lobe, it not only influences executive function, but also the middle areas of the frontal lobe. Therefore, patients with frontal meningioma developed more severe symptoms of apathy. Patients in the MF group also had more severe symptoms of apathy because frontal meningioma was near to the cingulate gyrus.

\section{Apathy and brain edema}

AES scores were much higher in patients with brain edema than in patients without brain edema, but the difference was only statistically significant in the MF group. Our explanation for this finding is that the tumors were much closer to the cingulate gyrus in the MF group had than in the other groups, so brain edema in the MF group could be associated with more severe apathy. Further, logistic regression analysis showed no relationship between brain edema and apathy, suggesting that brain edema alone might not be an important risk factor for apathy, but it might cause apathy if occurring in specific tumor location.

\section{Tumor removal and apathy}

AES scores at follow-up 3 months after surgery were much lower than those before surgery. Almost half of the patients with apathy returned to normal during the follow-up period. Previous studies have also demonstrated an improvement in symptoms of apathy after surgery in patients with frontal meningioma, ${ }^{7,11}$ suggesting that apathy caused by meningioma might be reversible. Other neural dysfunctions have also been reported to recover totally or partially after resection of meningioma. ${ }^{23,25,26}$ This may be related to certain features of meningioma. Unlike other brain tumors, most meningiomas do not invade neighboring brain tissue. Meningiomas are extracerebral tumors with an arachnoid space between the tumor and the cortex, so when the tumor is resected carefully, the neighboring neural structures remain intact. This allows the possibility 
for restoration of function in the affected neural structures, which is rare in most diseases encountered in neurology. It would therefore be feasible to do research on apathy before and after surgery, which would be almost impossible in neurological disorders like Alzheimer's disease and Parkinson's disease.

Further comparison of AES scores before and after surgery showed the greatest decreases in the MF group, followed by the VF group, suggesting that compression of the medial or ventral aspect of the frontal cortex is a major contributor to apathy. However, we did not find this association in the DLF and NF groups, suggesting that those regions might not be involved in apathy. However, we did find that apathy persisted in some MF patients after surgery, which might be attributed to incomplete recovery, suggesting that 3 months of follow-up after surgery might be too short to evaluate full recovery. Apoptosis of neurons and demyelination of white matter due to long-term ischemia might also need to be taken into consideration as a cause for lack of recovery of neural function.

\section{Study limitations}

A relatively small sample size was enrolled in this study, mainly because of time and funding constraints, and it might be insufficient to demonstrate the correlation between some minor factors, such as VF meningioma, and apathy statistically. This may also explain why the AES score was high in the VF group before surgery, yet the follow-up study did not show any statistically significant difference in AES scores before and after treatment in this group, and why MF but not VF entered the equation in logistic regression. The dropout rate during the follow-up period was high, mainly because most of the patients did not live in Shanghai. When recovered, most returned home and were either unwilling to come back for further study or were totally lost to contact. Further research should take these factors into consideration; higher proportion of locally resident subjects or an appropriate pecuniary reward might help to improve this situation. In addition, with regard to the imaging method used to detect the pathological changes associated with apathy, MRI was only able to show the location of the tumor and other indirect factors such as tumor size and edema in brain tissue. Future research should consider use of advanced techniques such as single-photon emission computed tomography or positron emission tomography, ${ }^{27}$ to show changes in metabolites or neurotransmitter-receptors, which may enrich our understanding of the mechanism of apathy.

\section{Acknowledgment}

This work was supported by the National Natural Science Foundation of China for Youth (81201033 to CS) and Shanghai Health Bureau Foundation (20114358 to CS).

\section{Disclosure}

The authors report no conflict of interest concerning the materials or methods used or the findings reported in this paper.

\section{References}

1. Marin RS. Apathy: a neuropsychiatric syndrome. J Neuropsychiatry Clin Neurosci. 1991;3(3):243-254.

2. Raffard S, Esposito F, Boulenger JP, Van der Linden M. Impaired ability to imagine future pleasant events is associated with apathy in schizophrenia. Psychiatry Res. 2013;209(3):393-400.

3. Robert PH, Mulin E, Mallea P, David R. Review: apathy diagnosis, assessment, and treatment in Alzheimer's disease. CNS Neurosci Ther. 2010; 16(5):263-271.

4. Robert G, Le Jeune F, Dondaine T, et al. Apathy and impaired emotional facial recognition networks overlap in Parkinson's disease: a PET study with conjunction analyses. J Neurol Neurosurg Psychiatry. 2014; 85(10): 1153-1158.

5. Caeiro L, Ferro JM, Figueira ML. Apathy in acute stroke patients. Eur J Neurol. 2012;19(2):291-297.

6. Starkstein SE, Pahissa J. Apathy following traumatic brain injury. Psychiatr Clin North Am. 2014;37(1):103-112.

7. Weitzner MA. Psychosocial and neuropsychiatric aspects of patients with primary brain tumors. Cancer Invest. 1999;17(4):285-291.

8. Schaller B. Influences of brain tumor-associated $\mathrm{pH}$ changes and hypoxia on epileptogenesis. Acta Neurol Scand. 2005;111(2):75-83.

9. Schaller BJ. Strategies for molecular imaging dementia and neurodegenerative diseases. Neuropsychiatr Dis Treat. 2008;4(3):585-612.

10. Starkstein SE, Mizrahi R, Capizzano AA, Acion L, Brockman S, PowerBD. Neuroimaging correlates of apathy and depression in Alzheimer's disease. J Neuropsychiatry Clin Neurosci. 2009;21(3):259-265.

11. Kim JW, Lee DY, Choo IH, et al. Microstructural alteration of the anterior cingulum is associated with apathy in Alzheimer disease. Am J Geriatr Psychiatry. 2011;19(7):644-653.

12. Mori T, Shimada H, Shinotoh H, et al. Apathy correlates with prefrontal amyloid beta deposition in Alzheimer's disease. J Neurol Neurosurg Psychiatry. 2014;85(4):449-455.

13. Cretin B, Echaniz-Laguna A, Meyer C, Blanc F, Sellal F. [Apathy or depression? Do you have a nose for it? Four case reports of paramedian frontal tumors]. Rev Neurol (Paris). 2010;166(8-9):704-710. French.

14. Thaler D, Chen YC, Nixon PD, Stern CE, Passingham RE. The functions of the medial premotor cortex. I. Simple learned movements. Exp Brain Res. 1995;102(3):445-460.

15. Kang SY, Kim JS. Anterior cerebral artery infarction: stroke mechanism and clinical-imaging study in 100 patients. Neurology. 2008;70(24 Pt 2): 2386-2393.

16. Narita M, Matsushima Y, Niikura K, et al. Implication of dopaminergic projection from the ventral tegmental area to the anterior cingulate cortex in mu-opioid-induced place preference. Addict Biol. 2010; 15(4):434-447.

17. Hayden BY, Platt ML. Neurons in anterior cingulate cortex multiplex information about reward and action. $J$ Neurosci. 2010;30(9): 3339-3346.

18. Salamone JD, Correa M, Farrar A, Mingote SM. Effort-related functions of nucleus accumbens dopamine and associated forebrain circuits. Psychopharmacology (Berl). 2007;191(3):461-482.

19. Bush G, Luu P, Posner MI. Cognitive and emotional influences in anterior cingulate cortex. Trends Cogn Sci. 2000;4(6):215-222. 
20. Hahn C, Lim HK, Won WY, Ahn KJ, Jung WS, Lee CU. Apathy and white matter integrity in Alzheimer's disease: a whole brain analysis with tract-based spatial statistics. PLoS One. 2013;8(1):e53493.

21. Yang SR, Shang XY, Tao J, Liu JY, Hua P. Voxel-based analysis of fractional anisotropy in post-stroke apathy. PLoS One. 2015;10(1): e116168.

22. Marin RS, Wilkosz PA. Disorders of diminished motivation. J Head Trauma Rehabil. 2005;20(4):377-388.

23. Bassiouni H, Asgari S, Stolke D. Olfactory groove meningiomas: functional outcome in a series treated microsurgically. Acta Neurochir (Wien). 2007;149(2):109-121.

24. Tahta K, Cirak B, Pakdemirli E, Suzer T, Tahta F. Postoperative mutism after removal of an anterior falcine meningioma. J Clin Neurosci. 2007;14(8):793-796.
25. Oyama H, Noda S, Negoro M, et al. Giant meningioma fed by the anterior choroidal artery: successful removal following embolization - case report. Neurol Med Chir (Tokyo). 1992;32(11):839-841.

26. Goto H, Nishizaki T, Kajiwara K, et al. Improvement in cognitive function after radical excision of an anterior skull base meningioma - a report of 2 cases. J Clin Neurosci. 2003;10(3):375-378.

27. Schaller B. Usefulness of positron emission tomography in diagnosis and treatment follow-up of brain tumors. Neurobiol Dis. 2004; 15(3):437-448.

\section{Publish your work in this journal}

Neuropsychiatric Disease and Treatment is an international, peerreviewed journal of clinical therapeutics and pharmacology focusing on concise rapid reporting of clinical or pre-clinical studies on a range of neuropsychiatric and neurological disorders. This journal is indexed on PubMed Central, the 'PsycINFO' database and CAS, and is the official journal of The International Neuropsychiatric Association (INA). The manuscript management system is completely online and includes a very quick and fair peer-review system, which is all easy to use. Visit http://www.dovepress.com/testimonials.php to read real quotes from published authors.

Submit your manuscript here: http://www.dovepress.com/neuropsychiatric-disease-and-treatment-journal 